Honam Mathematical J. 34 (2012), No. 2, pp. 191-198

http://dx.doi.org/10.5831/HMJ.2012.34.2.191

\title{
THE SECOND DERIVATIVE OF THE ENERGY FUNCTIONAL
}

\author{
HWAJEONG KIM
}

\begin{abstract}
Minimal surfaces with given boundaries are the solutions of Plateau's problem. In studying the calculus of variations for the minimal surfaces, the functional $\mathcal{E}$, corresponding to the energy of surfaces, is introduced in [Ki09]. In this paper we derive a formula for the second derivative of $\mathcal{E}$, which is necessary for further theories of the calculus of variations.
\end{abstract}

\section{Introduction}

The calculus of variations for minimal surfaces with given boundaries was one of the fascinating but very difficult problems in mathematics for a long time. In 1980's, studying the Ljusternik-Schnirelmann theory on convex sets in Banach spaces, M. Struwe proposed a variational approach to unstable minimal surfaces of disc or annulus type in $\mathbb{R}^{n}$ ([St1][St2][St3]).

And then by J. Hohrein ([Ho]) and H. Kim ([Ki07] [Ki09]) the theories for the minimal surfaces in Riemannian manifolds were developed. In these papers, a minimal surface with a given boundary is characterized as a critical point of a functional corresponding to the energy of surfaces. More precisely, for a parametrization $x$ of the given boundary, the functional $\mathcal{E}$ is defined by

$$
\mathcal{E}(x)=\frac{1}{2} \int|d \mathcal{F}(x)|^{2} d w,
$$

where $\mathcal{F}(x)$ is the harmonic extension with boundary parametrization $x$ of disc type or annulus type. The functional $\mathcal{E}$, defined on a proper set of maps, is then proved to be in the class of $C^{1}$.

Received February 9, 2012. Accepted February 24, 2012.

2000 Mathematics Subject Classification. 49Q05, 58E05.

Key words and phrases.Minimal surfaces, Plateau's problem.

Supported by Hannam University Research Fund in 2012. 
In Euclidean space, the harmonic extension operator $\mathcal{H}(x)$ is linear and since the energy functional is in $C^{\infty}$, the functional $\mathcal{E}$ is also in $C^{\infty}$. But in the Riemannian case, unfortunately the harmonic operator $\mathcal{F}(x)$ is not linear and the differentiability of $\mathcal{E}$ is not trivial. In fact, in [Ho] and [Ki09], the authors proved the $C^{1}$ - differentiability of $\mathcal{E}$ with many computations. For further interesting discussions in the calculus of variations, the higher differentiability of $\mathcal{E}$ is to need. Actually for the morse theory the $C^{2}$-differentiability of $\mathcal{E}$ is necessary.

In this article, we will discuss on the $C^{2}$-differentiability of $\varepsilon$.

\section{Setting and notations}

\subsection{Some definitions}

Let $(N, h)$ be a connected, oriented, complete Riemannian manifold of dimension $n \geq 2$ with metric $h$. Then $(N, h)$ can be embedded isometrically and properly into some $\mathbb{R}^{k}$ as a closed submanifold by means of the map $\eta$ ([Gro]).

- Let

$$
B:=\left\{z=\left(z^{1}, z^{2}\right) \in \mathbb{R}^{2}|| z \mid \leq 1\right\} .
$$

By $d \omega$ and $d_{0}$ we denote the area elements in $B$ and in $\partial B$ respectively.

- For $f \in C^{2}(B,(N, h)), d f$ is a section of $T^{*} B \otimes f^{*} T N$, i.e.

$$
d f=f_{, i}^{\alpha} d z^{i} \otimes \frac{\partial}{\partial y^{\alpha}} \circ f
$$

here we use the summation convention for indices and a colon denotes the ordinary derivative with $i=1,2, \alpha=1, \cdots, n$.

- In this section we also recall the definitions of the Sobolev spaces in $\mathbb{R}^{k}$ and in a Riemannian manifold (see [Ad] [St1]).

We define a Banach space as follows:

$$
H^{1,2} \cap C^{0}(B, N):=\left\{f \in H^{1,2} \cap C^{0}\left(B, \mathbb{R}^{k}\right) \mid f(B) \subset N\right\}
$$

with norm $\|f\|_{1,2 ; 0}:=\|d f\|_{L^{2}}+\|f\|_{C^{0}}$.

- For $f \in C^{1}(B, N), V=V^{\alpha} \frac{\partial}{\partial y^{\alpha}} \circ f \in C^{1}\left(B, f^{*} T N\right), \alpha=1, \cdots, n$ is called a vector field along $f$ with the covariant derivative along $f$ as follows:

$$
\nabla^{f} V:=\left(V_{, i}^{\alpha}+V^{\gamma} f_{, i}^{\beta} \Gamma_{\beta \gamma}^{\alpha}\right) d z^{i} \otimes \frac{\partial}{\partial y^{\alpha}} \circ f
$$


where $V_{, i}^{\alpha}$ means the ordinary differential of $V^{\alpha}$ with respect to the $z^{i}$ variable.

Now set

$T_{f} H^{1,2} \cap C^{0}(B, N) \cong\left\{V \in H^{1,2} \cap C^{0}\left(B, \mathbb{R}^{k}\right) \mid V(\cdot) \in T_{f(\cdot)} N\right\}=: H^{1,2} \cap$ $C^{0}\left(B, f^{*} T N\right)$, with norm

$$
\|V\|:=\left(\int_{B}\left|\nabla^{f} V\right|_{h}^{2} d \omega\right)^{\frac{1}{2}}+\|V\|_{C^{0}} \cong\left(\int_{B}|d V|_{\mathbb{R}^{k}}^{2} d \omega\right)^{\frac{1}{2}}+\|V\|_{C^{0}} .
$$

- Let $\Gamma$ be a Jordan curve in $N$ diffeomorphic to $S^{1}:=\partial B$. Then we define a Hilbert space

$$
H^{\frac{1}{2}, 2} \cap C^{0}(\partial B ; \Gamma):=\left\{u \in H^{\frac{1}{2}, 2} \cap C^{0}\left(\partial B, \mathbb{R}^{k}\right) \mid u(\partial B)=\Gamma\right\},
$$

where the norm is given by $\|u\|_{\frac{1}{2}, 2 ; 0}:=\|d \mathcal{H}(u)\|_{L^{2}}+\|u\|_{C^{0}}$, and $\mathcal{H}(u)$ is the harmonic extension in $\mathbb{R}^{k}$ with $\left.\mathcal{H}(u)\right|_{\partial B}(\cdot)=u(\cdot)$.

In addition, the tangent space of $u$ is given by

$$
\begin{aligned}
T_{u} H^{\frac{1}{2}, 2} \cap C^{0}(\partial B ; \Gamma) & :=\left\{\xi \in H^{\frac{1}{2}, 2} \cap C^{0}\left(\partial B, u^{*} T N\right) \mid \xi(z) \in T_{u(z)} \Gamma, \text { for all } z \in \partial B\right\} \\
& =H^{\frac{1}{2}, 2} \cap C^{0}\left(\partial B, u^{*} T \Gamma\right) .
\end{aligned}
$$

- The manifold $N$ can be equipped with another metric $\tilde{h}$ such that $\Gamma$ is a geodesic in $(N, \tilde{h})$. Then we observe that $H^{1,2} \cap C^{0}\left((B, \partial B),(N, \Gamma)_{\tilde{h}}\right)$ and $H^{1,2} \cap C^{0}\left((B, \partial B),(N, \Gamma)_{h}\right)$ coincide as sets. So, in the computation of the exponential map in $N$ we can use the metric $\tilde{h}$.

- The energy of $f \in H^{1,2}(\Omega, N)$ is denoted by

$$
E(f):=\frac{1}{2} \int_{\Omega}|d f|_{h}^{2} d w .
$$

- The Euler-Lagrange equation of $E$ for $f \in C^{2}(B,(N, h))$, called the tension field along $f$, is as follows:

$$
\begin{aligned}
\tau_{h}(f) & :=\left\langle\nabla_{\frac{\partial}{\partial z^{i}}} d f, d z^{i}\right\rangle=g^{i j}(\nabla d f)_{i j}^{\alpha} \\
& =g^{i j}\left(f_{, i j}^{\alpha}-f_{, k}^{\alpha} \Gamma_{i j}^{k}+f_{, i}^{\beta} f_{, j}^{\gamma} \Gamma_{\beta \gamma}^{\alpha} \circ f\right) \frac{\partial}{\partial y^{\alpha}} \circ f .
\end{aligned}
$$

And $f \in C^{2}(B,(N, h))$ is called harmonic if $\tau_{h}(f)=0$.

- Finally, the trace of a two form $R$ in $T B^{*}$ can be defined by

$$
\left.\operatorname{tr} R:=\left\langle(R\lrcorner \frac{\partial}{\partial z^{i}}\right), d z^{i}\right\rangle_{T B^{*}} .
$$




\subsection{Setting}

- We consider a Riemannian manifold $N$ and a boundary curve $\Gamma$ which satisfy $(\mathrm{C} 1)$ or $(\mathrm{C} 2)$ as follows.

(C1) There exists $p \in N$ with $\Gamma \subset B(p, r)$, where a geodesic ball $B(p, r)$ lies within the normal range of all its points. We assume $r<$ $\pi /(2 \sqrt{\kappa})$, where $\kappa$ is an upper bound of the sectional curvature of $(N, h)$.

(C2) $N$ is compact with nonpositive sectional curvature.

These conditions are related to the existence and the uniqueness of the harmonic extension for a given boundary map ([HKW]).

- We take a subset $\mathcal{M}$ of $H^{\frac{1}{2}, 2} \cap C^{0}(\partial B ; \Gamma)$ such that for each $x \in \mathcal{M}$ there exists a unique harmonic extension (in $N$ ) of disc type on $B$. For details we refer to [Ki09].

And for $x \in \mathcal{M}$,

$$
\mathcal{F}(x): B \rightarrow N
$$

means the unique harmonic extension with boundary map $x$ on $\partial B$.

- Then for each $x \in \mathcal{M}$, we define a variational set $\mathcal{T}_{x}$ as follows:

Let $\gamma: \partial B \rightarrow \Gamma$ be a $C^{\infty}$ diffeomorphism. Since $\partial B \cong \mathbb{R} / 2 \pi$, we can take a weakly monotone map $\hat{x} \in C^{0}(\mathbb{R}, \mathbb{R})$ with $\hat{x}(\theta+2 \pi)=\hat{x}(\theta)+2 \pi$, such that $x(\theta)=\gamma(\cos (\hat{x}(\theta)), \sin (\hat{x}(\theta)))=: \gamma \circ \hat{x}(\theta)$. In addition $\hat{x}=$ $\tilde{x}+I d$ for some $\tilde{x} \in C^{0}(\partial B, \mathbb{R})$.

We then consider

$W_{\mathbb{R}^{k}}:=\left\{w \in C^{0}(\mathbb{R}, \mathbb{R}) \mid w\right.$ is weakly monotone, $\left.w(\theta+2 \pi)=w(\theta)+2 \pi ; E(\mathcal{H}(\gamma \circ w))<\infty\right\}$.

Clearly, $W_{\mathbb{R}^{k}}$ is convex (for further details, refer to [St1]).

Now for $x \in \mathcal{M}$, we define

$$
\mathcal{T}_{x}=\left\{d \gamma\left((w-\hat{x}) \frac{d}{d \theta} \circ \tilde{x}\right) \mid w \in \hat{x}_{\mathbb{R}^{k}} \text { and } \gamma \circ \hat{x}=x\right\} .
$$

Note that $\mathcal{T}_{x}$ is convex in $T_{x} H^{\frac{1}{2}, 2} \cap C^{0}\left(\partial B ; \Gamma_{i}\right)$, since $W_{\mathbb{R}^{k}}$ is convex.

- Now we define

$$
\begin{aligned}
\mathcal{E}: & \mathcal{M} \\
x & \longmapsto \mathbb{R} \\
& \longmapsto E(\mathcal{F}(x)):=\frac{1}{2} \int_{B}|d \mathcal{F}(x)|_{h}^{2} d \omega,
\end{aligned}
$$

where $\mathcal{F}(x): B \rightarrow N$ be the unique harmonic extension in $N$ with $x$ on $\partial B$. 


\section{The differentiability of $\mathcal{E}$}

\section{1. $1^{\text {st }}$-differentiability of $\mathcal{F}$}

For $x \in \mathcal{M}$ and $\xi \in \mathcal{T}_{x}$, let $\mathcal{F}_{t}=\mathcal{F}(\exp (t \xi)) \in H^{1,2}(B, N), t \in(-\varepsilon, \varepsilon)$. Since $N$ is imbedded in an Euclidean $\mathbb{R}^{k}$, we calculate $\mathcal{F}_{t}-\mathcal{F}_{0}$ actually in $\mathbb{R}^{k}$. The first derivative of the harmonic extension operator $\mathcal{F}$ can be defined as follows: for $\xi \in H^{\frac{1}{2}, 2}\left(\partial B,(x)^{*} T \Gamma\right)$

$$
\lim _{t \rightarrow 0} \frac{\mathcal{F}_{t}-\mathcal{F}_{0}}{t}=D \mathcal{F}_{x}(\xi),
$$

and it turns out, $D \mathcal{F}_{x}(\xi)$ is the Jacobi field $\mathbf{J}_{0}(\xi)$ along $\mathcal{F}_{0}$ with a given boundary map $\xi$ on $\partial B$.

Now the first derivative of $\mathcal{E}$ at $x \in \mathcal{M}$ in direction $\xi \in \mathcal{T}_{x}$ can be computed as follows (see [Ki09]):

$$
\langle\delta \mathcal{E}, \xi\rangle:=\left.\frac{d}{d t}\right|_{t=0} \varepsilon(\exp (t \xi))=\int\left\langle d \mathcal{F}_{0}, \nabla \mathbf{J}_{0}(\xi)\right\rangle_{h} d w .
$$

\subsection{Higher differentiability of $\mathcal{E}$}

For $\xi, \psi \in \mathcal{T}_{x}$, we can define the second derivative by

$$
\begin{aligned}
\left\langle\delta^{2} \mathcal{E}, \psi, \xi\right\rangle & =\frac{\partial^{2}}{\partial t \partial s} E\left(\left.\mathcal{F}_{\rho}\left(\exp _{x^{1}}(t \xi+s \psi)\right)\right|_{t=s=0}\right. \\
& =\left.\frac{d}{d t}\right|_{t=0} \int\left\langle d \mathcal{F}\left(\exp _{x}(t \xi)\right), \nabla \mathbf{J}_{\mathcal{F}\left(\exp _{x}(t \xi)\right)}\left(d \exp _{x, t \xi}(\psi)\right)\right\rangle d w .
\end{aligned}
$$

Here $\mathbf{J}$ is the first derivative of the harmonic extension operator $\mathcal{F}$, so for the second differentiability of $\mathcal{E}$ we actually need the second derivative of $\mathcal{F}$.

In the Euclidean case, the differentiability of the harmonic extension is easy, because of the linearity of the harmonic extension operator and the definition of the norm of the boundary map, that is the $H^{\frac{1}{2}, 2}$-norm (see [St2]). But in a Riemannian manifold, the harmonic extension operator is not linear, furthermore the natural candidate of the second derivative of $\mathcal{F}$.

$$
\lim _{t \rightarrow 0} \frac{\mathbf{J}_{\mathcal{F}\left(\exp _{x}(t \xi)\right)}\left(d \exp _{x, t \xi}(\psi)\right)-\mathbf{J}_{\mathcal{F}(x)}(\xi)}{t},
$$

which can be calculated $\mathbb{R}^{k}$, is not necessarily a map from $\partial B$ into $\mathcal{F}^{*} T N$, differently from the case of the first derivative of $\mathcal{F}$ (see (2)). Therefore, we need to analyze and understand the second derivative of $\mathcal{F}$ for the 
higher differentiability of that. In [Ki12], the second differentiability of $\mathcal{F}$ is discussed.

In this paper, we assume the second differentiability of $\mathcal{F}$ and compute the second derivative of $\mathcal{E}$. We will see that actually in the second derivative form of $\mathcal{E}$ we don't need the formula of the second derivative of $\mathcal{F}$. So, only the second differentiability of $\mathcal{F}$ is enough.

Theorem 3.1. Let $x \in \mathcal{M}$ be a critical point on $\mathcal{E}$. Then the second derivative of $\mathcal{E}$ at $x$ in directions $\xi, \psi \in \mathcal{T}_{x}$ is as follows:

$$
\begin{aligned}
\left\langle\delta^{2} \mathcal{E}, \xi, \psi\right\rangle= & \int_{B}\left\langle\nabla \mathbf{J}_{0}(\psi), \nabla \mathbf{J}_{0}(\xi)\right\rangle_{T^{*} B \otimes \mathcal{F}_{0}^{-1} T N} d \omega \\
& +\int_{B} \operatorname{tr}\left\langle R\left(\mathbf{J}_{0}(\psi), d \mathcal{F}_{0}\right) \mathbf{J}_{0}(\xi), d \mathcal{F}_{0}\right\rangle_{\mathcal{F}_{0}^{-1} T N} d \omega \\
& +\int_{\partial B}\left\langle d^{2} \gamma(w) \hat{\psi} \hat{\xi}, \frac{\partial \mathcal{F}_{0}}{\partial \vec{n}}\right\rangle_{h} d_{0},
\end{aligned}
$$

where we let $\vec{n}$ denote the outer normal to $\partial B$.

Proof. i) Let $\xi, \psi \in \mathcal{T}_{x}$. If $t, s \leq \frac{1}{2},(t+s) \xi,(t+s) \psi \in \mathcal{T}_{x}$ and

$$
t \xi+s \psi=\frac{t}{t+s}(t+s) \xi+\frac{s}{t+s}(t+s) \psi \in \mathcal{T}_{x},
$$

since $\mathcal{T}_{x}$ is defined to be convex.

Furthermore, there exist $t_{0}>0$ and $s_{0}>0$ such that $\exp _{x}(t \xi+s \psi) \in$ $M$ for all $t \in\left[0, t_{0}\right], s \in\left[0, s_{0}\right]$ by Lemma 2.2 in [Ki]. Then we define

$$
\delta^{2} \mathcal{E}(\xi, \psi)=\frac{\partial^{2}}{\partial t \partial s} E\left(\left.\mathcal{F}\left(\exp _{x}(t \xi+s \psi)\right)\right|_{t=s=0} .\right.
$$

ii) Denoting $\mathcal{F}\left(\exp _{x}(t \xi+s \psi), x^{2}\right)$ by $\mathcal{F}_{t s}$, we have, because the LeviCivita connection is metric and torsion free,

$$
\begin{aligned}
& \frac{\partial^{2}}{\partial t \partial s} \frac{1}{2}\left\langle\frac{\partial \mathcal{F}_{t s}}{\partial z^{i}} d z^{i}, \frac{\partial \mathcal{F}_{t s}}{\partial z^{j}} d z^{j}\right\rangle_{T B^{*} \mathcal{F}_{t s}^{-1} T N} \\
&=\frac{\partial}{\partial t}\left\langle\nabla_{\frac{\partial}{\partial z^{i}}} \frac{\partial \mathcal{F}_{t s}}{\partial s} d z^{i}, \frac{\partial \mathcal{F}_{t s}}{\partial z^{j}} d z^{j}\right\rangle_{T B^{*} \otimes \mathcal{F}_{t s}^{-1} T N} \\
&=\left\langle\nabla_{\frac{\partial}{\partial t}} \nabla_{\frac{\partial}{\partial z^{i}}} \frac{\partial \mathcal{F}_{t s}}{\partial s} d z^{i}, \frac{\partial \mathcal{F}_{t s}}{\partial z^{j}} d z^{j}\right\rangle+\left\langle\nabla_{\frac{\partial}{\partial z^{i}}} \frac{\partial \mathcal{F}_{t s}}{\partial s} d z^{i}, \nabla_{\frac{\partial}{\partial t}} \frac{\partial \mathcal{F}_{t s}}{\partial z^{j}} d z^{j}\right\rangle \\
&=\left\langle\nabla_{\frac{\partial}{\partial z^{i}}} \nabla_{\frac{\partial}{\partial t}} \frac{\partial \mathcal{F}_{t s}}{\partial s} d z^{i}, \frac{\partial \mathcal{F}_{t s}}{\partial z^{j}} d z^{j}\right\rangle+\operatorname{tr}\left\langle R\left(\frac{\partial \mathcal{F}_{t s}}{\partial t}, d \mathcal{F}_{t s}\right) \frac{\partial \mathcal{F}_{t s}}{\partial s}, d \mathcal{F}_{t s}\right\rangle_{\mathcal{F}_{t s}^{-1} T N} \\
&+\left\langle\nabla_{\frac{\partial}{\partial z^{i}}} \frac{\partial \mathcal{F}_{t s}}{\partial s} d z^{i}, \nabla_{\frac{\partial}{\partial z^{j}}} \frac{\partial \mathcal{F}_{t s}}{\partial t} d z^{j}\right\rangle,
\end{aligned}
$$


where $\left.R\left(\frac{\partial \mathcal{F}_{t s}}{\partial t}, d \mathcal{F}_{t s}\right) \frac{\partial \mathcal{F}_{t s}}{\partial s}, d \mathcal{F}_{t s}\right\rangle$ is the curvature tensor in $N$ but in the form of two form in $T B^{*}$. We also recall the definition of the trace of two forms (1).

We know that $\left.\frac{\partial \mathcal{F} t s}{\partial t}\right|_{t=s=0}=\mathbf{J}_{0}(\xi, 0)$ and $\left.\frac{\partial \mathcal{F} t s}{\partial s}\right|_{t=s=0}=\mathbf{J}_{0}(\psi, 0)$. Hence

$$
\begin{aligned}
\delta^{2} \mathcal{E}(\xi, \psi) & =\left.\int \frac{\partial^{2}}{\partial t \partial s}\right|_{t=s=0} E\left(\mathcal{F}_{t s}\right) d \omega \\
= & \int_{B}\left\langle\nabla \mathbf{J}_{0}(\xi, 0), \nabla \mathbf{J}_{0}(\psi, 0)\right\rangle_{T^{*} B \otimes \mathcal{F}_{0}^{-1} T N} d \omega \\
& +\int_{B} \operatorname{tr}\left\langle R\left(\mathbf{J}_{0}(\xi, 0), d \mathcal{F}_{\rho}\right) \mathbf{J}_{0}(\psi, 0), d \mathcal{F}_{0}\right\rangle_{\mathcal{F}_{0}^{-1} T N} d \omega \\
& +\left.\int_{B}\left\langle\nabla_{\frac{\partial}{\partial z^{i}}} \nabla_{\frac{\partial}{\partial t}} \frac{\partial \mathcal{F}_{t s}}{\partial s} d z^{i}, \frac{\partial \mathcal{F}_{t s}}{\partial z^{j}} d z^{j}\right\rangle\right|_{t=s=0} d \omega .
\end{aligned}
$$

We compute further the third term:

$$
\begin{aligned}
&\left.\int_{B}\left\langle\nabla_{\frac{\partial}{\partial z^{i}}} \nabla_{\frac{\partial}{\partial t}} \frac{\partial \mathcal{F}_{t s}}{\partial s} d z^{i}, \frac{\partial \mathcal{F}_{t s}}{\partial z^{j}} d z^{j}\right\rangle\right|_{t=s=0} d \omega \\
&=\left.\int_{B} \frac{\partial}{\partial z^{i}}\left\langle\nabla_{\frac{\partial}{\partial t}} \frac{\partial \mathcal{F}_{t s}}{\partial s} d z^{i}, \frac{\partial \mathcal{F}_{t s}}{\partial z^{j}} d z^{j}\right\rangle\right|_{t=s=0} d \omega \\
&-\left.\int_{B}\left\langle\nabla_{\frac{\partial}{\partial t}} \frac{\partial \mathcal{F}_{t s}}{\partial s} d z^{i}, \nabla_{\frac{\partial}{\partial z^{i}}} \frac{\partial \mathcal{F}_{t s}}{\partial z^{j}} d z^{j}\right\rangle\right|_{t=s=0} d \omega
\end{aligned}
$$

But the second term is zero, since $\mathcal{F}_{t s}$ are harmonic, so

$$
\nabla_{\frac{\partial}{\partial z^{i}}} \frac{\partial \mathcal{F}_{t s}}{\partial z^{j}}\left\langle d z^{j}, d z^{i}\right\rangle=\operatorname{tr} \nabla d \mathcal{F}_{t s}=0
$$

Meanwhile, from the divergence theorem in $\mathbb{R}^{2}$,

$$
\begin{aligned}
\int_{B} \frac{\partial}{\partial z^{i}}\langle & \left.\nabla_{\frac{\partial}{\partial t}} \frac{\partial \mathcal{F}_{t s}}{\partial s} d z^{i}, \frac{\partial \mathcal{F}_{t s}}{\partial z^{j}} d z^{j}\right\rangle\left.\right|_{t=s=0} d \omega \\
& =\left.\int_{B} \frac{\partial}{\partial z^{i}}\left\langle\nabla_{\frac{\partial}{\partial t}} \frac{\partial \mathcal{F}_{t s}}{\partial s}, \frac{\partial \mathcal{F}_{t s}}{\partial z^{i}}\right\rangle\right|_{t=s=0} d \omega \\
& =\int_{\partial B}\left\langle\left.\nabla_{\frac{\partial}{\partial t}} \frac{\partial \mathcal{F}_{t s}}{\partial s}\right|_{t=s=0}, \frac{\partial \mathcal{F}_{0}}{\partial \vec{n}}\right\rangle d_{0} \\
& =\int_{\partial B}\left\langle d^{2} \gamma \hat{\xi} \hat{\psi}, \frac{\partial \mathcal{F}_{0}}{\partial \vec{n}}\right\rangle_{h} d_{0},
\end{aligned}
$$

where $d \gamma(\hat{\xi})=\xi, d \gamma(\hat{\psi})=\psi$, and $\gamma \circ w=x$, so that

$$
\left.\nabla_{\frac{\partial}{\partial t}} \frac{\partial \mathcal{F}_{t s}}{\partial s}\right|_{\partial B}=\frac{\partial^{2}}{\partial t \partial s}\left(\exp _{x}(t \xi+s \psi)\right)=\frac{\partial^{2}}{\partial t \partial s} \gamma(w+t \hat{\xi}+s \hat{\psi}) .
$$


We note, since $x$ is a critical point of $\mathcal{E}$ and $\mathcal{F}_{0}$ is in the class os $H^{2,2}(B)$ $([\mathrm{Ki} 07])$. And a Sobolev embedding theorem implies, $\frac{\partial \mathcal{F}_{0}}{\partial \vec{n}}$ is well defined on $\partial B$.

Hence we have the above formula (4) of the second derivative of $\mathcal{E}$.

In the Euclidean case, where the curvature tensor $R$ vanishes, the formula (4) implies the well known formula in [St2].

\title{
References
}

[Ad] Adams, R.A.: Sobolev Spaces Academic Press, New York - San Francisco London, 1975.

[Gro] Gromov, M.L., Rohlin, V.A.:, Imbeddings and immersion in Riemannian geometry, Russ. Math. Surveys 25 (1970), 1-57.

[HH] Heinz, E., Hildebrandt, S.:, Some Remarks on Minimal Surfaces in Riemannian Manifolds, Communications on Pure and Applied mathematics, vol.XXIII (1970), 371-377.

[HKW] Hildebrandt, S., Kaul, H., Widman, K.O.:, An existence theorem for harmonic mappings of Riemannian manifolds, Acta Math.138 (1977), 1-16.

[Ho] Hohrein, J.:, Existence of unstable minimal surfaces of higher genus in manifolds of nonpositive curvature Dissertation, 1994.

[Ki] Kim, H. :, Unstable minimal surfaces of annulus type in manifolds, Dissertation 2004

[Ki07] - -:, A variational approach to the regularity of the minimal surfaces of annulus type in Riemannian manifolds, Differ. Geom. Appl. 25 (2007) 466-484.

[Ki09] - - , Unstable minimal surfaces of annulus type in manifolds, Adv. Geom. (2009), Issue 3, $401-436$.

[Ki12] - : , The second derivative of the harmonic extension operator in a Riemannian manifold., Preprint (2012).

[St1] Struwe, M.:, Plateau's Problem and the calculus of variations, Princeton U.P. 1998.

[St2] Struwe, M.:, A critical point theory for minmal surfaces spanning a wire in $\mathbb{R}^{k}$, J.reine angew. Math. 349 (1984) 1-23.

[St3] Struwe, M.:, A Morse Teory for annulus type minimal surfaces, J. Reine u. Angew. Math. 368 (1986), 1-27.

\author{
Hwajeong Kim \\ Department of Mathematics, Hannam University, \\ Daejeon 306-791, Korea. \\ E-mail: hwajkim@hnu.kr
}

